\title{
Dissociation of within- and between-Session Extinction of Conditioned Fear
}

\author{
Wolfgang Plendl and Carsten T. Wotjak \\ Max Planck Institute of Psychiatry, 80804 Munich, Germany
}

Recent findings obtained in patients with phobias or trauma-related anxiety disorders raise doubts concerning the interrelation between acute fear relief during an exposure-based therapeutic session and beneficial treatment progress. In a mouse model explicit for exposure therapy, we challenge the view that within-session fear reduction is the turning point for relearning of a stimulus-threat association. Even though within-session extinction of auditory-cued fear memory was identical for prolonged and spaced tone presentations, only the latter caused between-session extinction. Furthermore, spaced tone presentations led to between-session extinction even in the complete absence of within-session extinction, as observed for remote fear memories and in case of abolished cannabinoid receptor type 1 signaling. Induction of between-session extinction was accompanied by an increase in the number of c-Fos-positive neurons within the basolateral amygdala, the cingulate cortex, and the dentate gyrus, independent of the level of within-session extinction. Together, our findings demonstrate that within-session extinction is neither sufficient nor essential for between-session extinction, thus calling for a reconsideration of current concepts underlying exposure-based therapies.

\section{Introduction}

Exposure therapy is an effective treatment of phobias and anxiety disorders (Marks and Tobena, 1990; Grös and Antony, 2006; Norton and Price, 2007). Prevailing models interpret the level of fear throughout exposure trials as an index of beneficial learning. It is generally assumed that, during an exposure session, a substantial amount of fear has to be elicited (also called "initial fear activation") and subsequently to wane (in a process called "within-session habituation") to achieve lasting fear alleviation (i.e., between-session habituation) (Craske et al., 2008). Recent findings challenge this view, indicating that performance during training may not be proportional to therapeutic progress (Pitman et al.,1996a,b; Craske et al., 2008).

Extinction of conditioned fear is the laboratory analog of exposure therapy in rodents (Rosen and Schulkin, 1998; Myers and Davis, 2002, 2007; Barad, 2005). Conditioned fear is induced by pairing a neutral environmental stimulus [conditioned stimlus (CS), e.g., tone] with an aversive outcome [unconditioned stimulus (US), e.g., electric footshock]. Re-exposure to the CS elicits a stereotypic, reproducible, and quantifiable behavioral response (conditioned response, e.g., freezing). During extinction training, repeated CS presentations in the absence of the US lead to a decay of the fear response (McSweeney and Swindell, 2002). Ac-

Received Dec. 7, 2009; revised Jan. 18, 2010; accepted Feb. 12, 2010.

This study was supported in part by grants from the Huebner Foundation and the Deutsch-Franzoesische Hochschule (to C.T.W.). We thank Beat Lutz (University of Mainz, Germany) and Giovanni Marsicano (INSERM Bordeaux, France) for generously providing (B1-deficient mice, Mario Engelmann (University of Magdeburg, Germany) for his advice concerning c-Fos immunohistochemistry and Isabella Braun for the help with its establishment, and Kornelia Kamprath (University of Münster, Germany) and Anja Siegmund (Charité Berlin, Germany) for their valuable comments on an earlier version of this manuscript.

Correspondence should be addressed to Dr. Carsten T. Wotjak, Max Planck Institute of Psychiatry, Kraepelinstrasse 2-10, 80804 Munich, Germany. E-mail: wotjak@mpipsykl.mpg.de.

D01:10.1523/JNEUROSCI.6038-09.2010

Copyright $\odot 2010$ the authors $\quad 0270-6474 / 10 / 304990-09 \$ 15.00 / 0$ cording to current theories, this decrease does not result from temporal decay or degradation of the original excitatory memory trace but is due to an inhibitory memory trace, which is built up on re-exposure to the CS in the absence of the US. This inhibitory memory trace (CS-no US) competes with and/or suppresses the original excitatory CS-US memory trace. This notion is supported by spontaneous recovery of the fear response with the passage of time after completion of extinction training and its renewal in an environment different from the extinction context (Myers and Davis, 2002, 2007; Ehrlich et al., 2009).

We recently demonstrated that the decrease of conditioned fear over the course of a prolonged tone presentation resembles habituation-like processes (Kamprath and Wotjak, 2004), which crucially involve cannabinoid receptor type 1 (CB1) signaling (Kamprath et al., 2006, 2009). Also with this modality, CB1deficient mice were strongly impaired, not only in within-session extinction, but also in between-session extinction of aversive memories (Marsicano et al., 2002). These phenotypes support the notion of a causal relationship of within-session (performance) and between-session (progress) extinction.

Analysis of the mechanisms underlying extinction learning and the resulting conditions that facilitate or impair extinction may help to unravel the relationship between extinction performance and extinction progress and to refine exposure-based therapies. Therefore, the present study tried to dissect withinsession and between-session extinction in mice with or without intact CB1 signaling in extinction paradigms with different exposure modalities. We provide evidence that spaced extinction trials are most efficient in eliciting between-session extinction. Moreover within-session extinction turned out to be neither sufficient nor essential for between-session extinction. In addition, we demonstrate that CB1 signaling controls within-session extinction but is largely dispensable for between-session extinction. By 
combining these results, we identified anatomical signatures of extinction training.

\section{Materials and Methods \\ Animals}

We used a total of 118 male $\mathrm{C} 57 \mathrm{BL} / 6 \mathrm{NCrl}$ (B6N purchased from Charles River Deutschland) and 28 male $\mathrm{CB} 1$ receptor-deficient $\left(\mathrm{CB} 1^{-1-}\right)$ mice, and 28 male wild-type littermates $\left(\mathrm{CB} 1^{+/+}\right.$; sample sizes for the individual experiments are given in the figure legends). Mutant mice and littermate controls were generated/genotyped as described before (Marsicano et al., 2002) and originated from our institutional breeding stock, which had been backcrossed to the B6N strain for six generations. At an age of 6-7 weeks, mice were separated and housed individually with food and water ad libitum under an inverse 12:12 h dark/light cycle (light off at 9:00 A.M.). Experiments were performed at an age of 9-12 weeks.

\section{Behavioral procedures}

All experimental procedures were approved by the Committee on Animal Health and Care of the State of Bavaria (Regierung von Oberbayern) and performed in strict compliance with the European Union recommendations for the care and use of laboratory animals (86/609/ CEE). Experiments were performed during the activity phase (i.e., dark phase) of the animals between 9:30 A.M. and 5:00 P.M. Animals of a given experiment derived from the same batch of mice and were tested simultaneously.

Fear conditioning. For conditioning, mice were placed in the conditioning context (Kamprath and Wotjak, 2004). Three minutes later, a tone ( $80 \mathrm{~dB}, 9 \mathrm{kHz}$ sine wave, $10 \mathrm{~ms}$ rising and falling time) was presented to the animals for $20 \mathrm{~s}$ that coterminated with a $2 \mathrm{~s}$ scrambled electric footshock of $0.7 \mathrm{~mA}$. Mice were returned to their home cages (hc) $60 \mathrm{~s}$ later.

Extinction training. Extinction procedures were adapted from Kamprath and Wotjak (2004) for permanent tone procedure, and from Herry et al. (2006) for variable interval procedure. Briefly, mice were placed in the test context, which differed from the conditioning context in material, shape, surface texture, and odor of the cleaning solution. After an initial $3 \mathrm{~min}$ of habituation, they were confronted either with a permanent $200 \mathrm{~s}$ tone $[9 \mathrm{kHz}, 80 \mathrm{~dB}$, sine-wave; permanent tone $(\mathrm{pt})]$ or with ten $20 \mathrm{~s}$ tones of the same characteristics presented with either constant intertone intervals (ci) of $20 \mathrm{~s}$ or variable tone intervals (vi) ranging from 20 to $180 \mathrm{~s}$ (mean, $80 \mathrm{~s}$ ). Mice returned to their hc $60 \mathrm{~s}$ after the end of the exposure protocol (see also Fig. $2 \mathrm{~A}$ ).

\section{Experiments}

Experiment 1. B6N mice were conditioned at day 0 (d0) and randomly assigned to one of three groups, which differed in extinction procedures at day 1 and day 2. Group I was exposed to a single pt, group II to ten $20 \mathrm{~s}$ tones with ci, and group III to ten $20 \mathrm{~s}$ tones with vi per day. At day 3 , all animals were exposed to a single $20 \mathrm{~s}$ tone (for details, see Fig. $2 \mathrm{~A}$ ).

Experiment 2 . B6N animals were conditioned at day 0 and randomly assigned to one of two groups, which differed in extinction procedures at day 40 and day 41 . Group I was exposed to a single pt, group II to ten $20 \mathrm{~s}$ tones with vi. At day 42, all animals were exposed to a single $20 \mathrm{~s}$ tone.

Experiment 3. For the first part of the experiment, $\mathrm{CB} 1^{-1-}$ and $\mathrm{CB} 1^{+/+}$mice were conditioned at day 0 and were exposed to a single $200 \mathrm{~s}$ tone per day (pt) on days 1, 2, 3, 10, and d40. For the second part, $\mathrm{B} 6 \mathrm{~N}$ mice were conditioned at $\mathrm{d} 0$ and randomly assigned to one of two groups. One group was treated with vehicle (Veh), the other with the CB1 receptor antagonist SR141716 (SR; $3 \mathrm{mg} / \mathrm{kg}$, s.c.) $1 \mathrm{~h}$ before exposure to a single $200 \mathrm{~s}$ tone (pt) on days 1, 2, 3, 10, and 40 .

Experiment 4. For the first part of the experiment, $\mathrm{CB} 1^{-1-}$ and $\mathrm{CB} 1^{+/+}$mice were conditioned at day 0 and exposed to ten $20 \mathrm{~s}$ tones with vi on days $1,2,3,10$, and 40 . For the second part, $\mathrm{B} 6 \mathrm{~N}$ mice were conditioned at day 0 and randomly assigned to one of two groups. One group was treated with Veh, the other with the CB1 receptor antagonist SR ( $3 \mathrm{mg} / \mathrm{kg}$, s.c.) $1 \mathrm{~h}$ before exposure to ten $20 \mathrm{~s}$ tones with vi on days 1 , 2, 3, 10, and 40 .

Experiment 5. B6N mice were conditioned at day 0 and randomly assigned to one of six groups, which differed in extinction procedures
Brain atlas figure

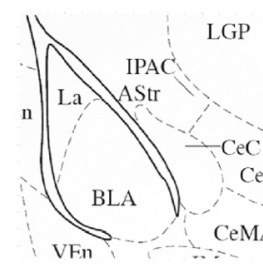

Bregma: $-0.94-{ }_{100}$ dark field + brain atlas

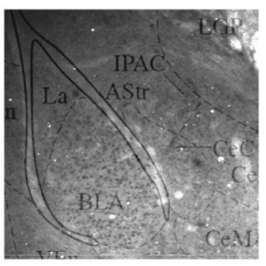

bright field + brain atlas

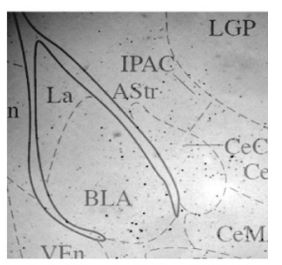

Figure 1. Localization of c-Fos-positive cells in the regions of interest. As shown exemplarily for the basolateral and central amygdala complex, ROls were selected according to the mouse brain atlas (Franklin and Paxinos, 1997). Dark-field and bright-field pictures of the same slide were taken and overlaid with brain atlas figures to visualize the boundaries of the ROls together with the c-Fos-positive cells.

and drug treatment. Injection took place $1 \mathrm{~h}$ before exposure on day 1 , when group I received a single $200 \mathrm{~s}$ tone (pt) after Veh injection, group II a single $200 \mathrm{~s}$ tone (pt) after SR injection ( $3 \mathrm{mg} / \mathrm{kg}$, s.c.), group III ten 20 s tones with vi after Veh injection, and group IV ten 20 s tones with vi after SR injection. Groups V and VI also received Veh and SR injections, respectively, but were left in their hc. All mice were processed for c-Fos immunohistochemistry $70 \mathrm{~min}$ after the end of extinction training (or the respective time in the home cage).

\section{Behavioral analysis}

The behavior of the mice was videotaped and scored off-line by a trained observer who was blind to the animals' treatment/genotype. Freezing was defined as the absence of all movements, except for those related to respiration.

\section{c-Fos immunohistochemistry}

Mice were deeply anesthetized with a mixture of ketamine/xylazine and transcardially perfused. Brains were removed, postfixed in $4 \%$ formaldehyde diluted in PBS for $20 \mathrm{~h}$ and transferred to $1 \mathrm{~m}$ sucrose in PBS for another $20 \mathrm{~h}$. The brains were shock frozen in isobutanol and stored at $-80^{\circ} \mathrm{C}$ until cryosectioning. Floating frontal sections $(40 \mu \mathrm{m})$ were incubated with an antibody raised against a peptide mapping at the $\mathrm{N}$ terminus of human c-Fos p62 (identical to the corresponding mouse sequence; c-Fos sc-42; 1:20000; Santa Cruz Biotechnology) for 4 d. c-Fosimmunoreactive cells were visualized using a biotinylated goat antirabbit secondary antibody (1:2000, Jackson Laboratory) and the ABC method (Richter et al., 2005). The number of c-Fos-immunoreactive cells was determined using a Zeiss microscope (Axiophot) and a computer program (Imagej; http://rsbweb.nih.gov/ij/). The following brain areas were analyzed: dentate gyrus (DG) and CA3 regions of the dorsal hippocampus, basolateral amygdala (BLA), lateral amygdala (LA), central amygdala $(\mathrm{CeA})$, cingulate cortex $(\mathrm{Cg} 1)$, prelimbic cortex $(\mathrm{PrL})$, and infralimbic cortex (IL) (Franklin and Paxinos, 1997) (see Fig. 6D). The total number of c-Fos-positive cells was counted within the regions of interest (ROIs) in both hemispheres on one representative section per mouse and ROI. Sections and ROIs were identified by combining darkfield pictures, bright-field pictures, and schematic drawings from a mouse brain atlas (Franklin and Paxinos, 1997) (for details, see Fig. 1). Sections were chosen and analyses performed unaware of the experimental history. The specificity of the staining procedure was confirmed by omission of the primary antibody (data not shown).

\section{Drug treatment}

SR141716 (Rimonabant) (Rinaldi-Carmona et al., 1994), kindly provided by the National Institute of Mental Health Chemical Synthesis and Drug Supply Program, was dissolved in vehicle solution (2.5\% DMSO and 1 drop of Tween 80 per $3 \mathrm{ml}$ of saline) and injected subcutaneously at $3 \mathrm{mg} / \mathrm{kg}$ body weight $1 \mathrm{~h}$ before each extinction training. For all groups, injections were given under light isoflurane anesthesia to avoid differences in coping with the stressful injection procedure between mice with intact and abolished CB1 signaling. 


\section{Statistical analysis}

Freezing behavior was analyzed in $20 \mathrm{~s}$ intervals and expressed as a percentage of the respective analysis interval. The following measures were considered for describing within- and between-session extinction: (1) changes in the total freezing response to the tone(s) over the course of the extinction training days (traditionally used measure of between-session extinction), (2) changes in freezing over the course of tone presentation(s) per day (in $20 \mathrm{~s}$ intervals; within-session extinction), and (3) changes in the initial freezing response to the first $20 \mathrm{~s}$ of tone presentation per day (defined by the length of the CS during conditioning) over the course of the extinction training days (newly suggested measure of between-session extinction). Data were analyzed by one-way ANOVAs for repeated measures (time), two-way ANOVAs (protocol/genotype/ treatment, time) for repeated measures (time $=$ interval) separately per test day (analysis of the freezing data in 20 s intervals), or two-way ANOVAs (protocol/genotype/treatment, time) for repeated measures (time $=$ analysis of the development of the total freezing responses/the initial freezing responses shown during the first $20 \mathrm{~s}$ of tone presentation over the course of the repeated training/testing days). c-Fos data were analyzed by two-way ANOVA (protocol, treatment) separately for each brain structure. Post hoc comparisons were performed by the NewmanKeuls test, if appropriate. Statistical significance was accepted if $p \leq$ 0.05 . Statistical analyses were performed using specialized software (GraphPad Prism 5.0, StatSoft Statistica 5.0, and SPSS version 16.0).

\section{Results}

\section{Within-session extinction is not sufficient for initiating} between-session extinction of recent fear memories

To study the interrelation of within- and between-session extinction, we conditioned male B6N mice with a single pairing of a $20 \mathrm{~s}$ tone with a footshock. The next 2 days, mice were re-exposed to the tone for a total of $200 \mathrm{~s}$ per day. Tones were presented either as a single $200 \mathrm{~s}$ pt or as series of $1020 \mathrm{~s}$ tones with ci or vi (Fig. $2 \mathrm{~A}$ ). All mice were exposed to a single $20 \mathrm{~s}$ tone at day 3 . All three groups showed the same decline in freezing on day 1 of extinction training (time: $F_{(9,243)}=22.5, p<0.001$ ), independently of the protocol (protocol: $F_{(2,27)}=0.81, p=0.921$; protocol $\times$ time: $F_{(18,243)}=0.71, p=0.789$ ) (Fig. $2 C$ ). Despite these similarities in within-session extinction, groups differed significantly in between-session extinction (protocol $\times$ time: $F_{(2,27)}=6.37, p=$ 0.005 ) (Fig. $2 B$ ) and in the development of initial freezing responses (protocol $\times$ time: $\left.F_{(4,54)}=6.91, p<0.001\right)($ Fig. $2 D)$. Post hoc analyses revealed that between-session decline in freezing was most pronounced in mice with repeated daily tone presentations at variable intervals, significantly retarded following tone presentations at constant intervals, and virtually absent in the case of permanent tone presentations. These data indicate that the predictability of tone presentations retards betweensession extinction and that within-session extinction per se is not sufficient for initiating between-session extinction.

\section{Within-session extinction is not essential for initiating between-session extinction of remote fear memories}

To test whether our conclusions also apply to extinction of remote fear memories, we conditioned male $\mathrm{B} 6 \mathrm{~N}$ mice with a single tone-shock pairing at day 0 and exposed them either to a single $\mathrm{pt}$ of $200 \mathrm{~s}$ or to series of $1020 \mathrm{~s}$ tone with vi at day 40 and day 41 after conditioning, followed by exposure to a single $20 \mathrm{~s}$ tone at day 42 . Both groups showed a similar nondecaying freezing response at day 40 (protocol: $F_{(1,14)}=0.39, p=0.845$; time: $F_{(9,126)}=$ $1.25, p=0.269$; protocol $\times$ time: $\left.F_{(9,126)}=1.01, p=0.428\right)$ (Fig. $3 B)$. At days 41 and 42 , however, mice of the vi group showed significantly less freezing than mice of the pt group $(p<0.005)$. This was reflected by reduced total freezing (protocol $\times$ time: $F_{(1,14)}=13.96, p=0.002$ ) (Fig. $3 A$ ) and significantly decreased

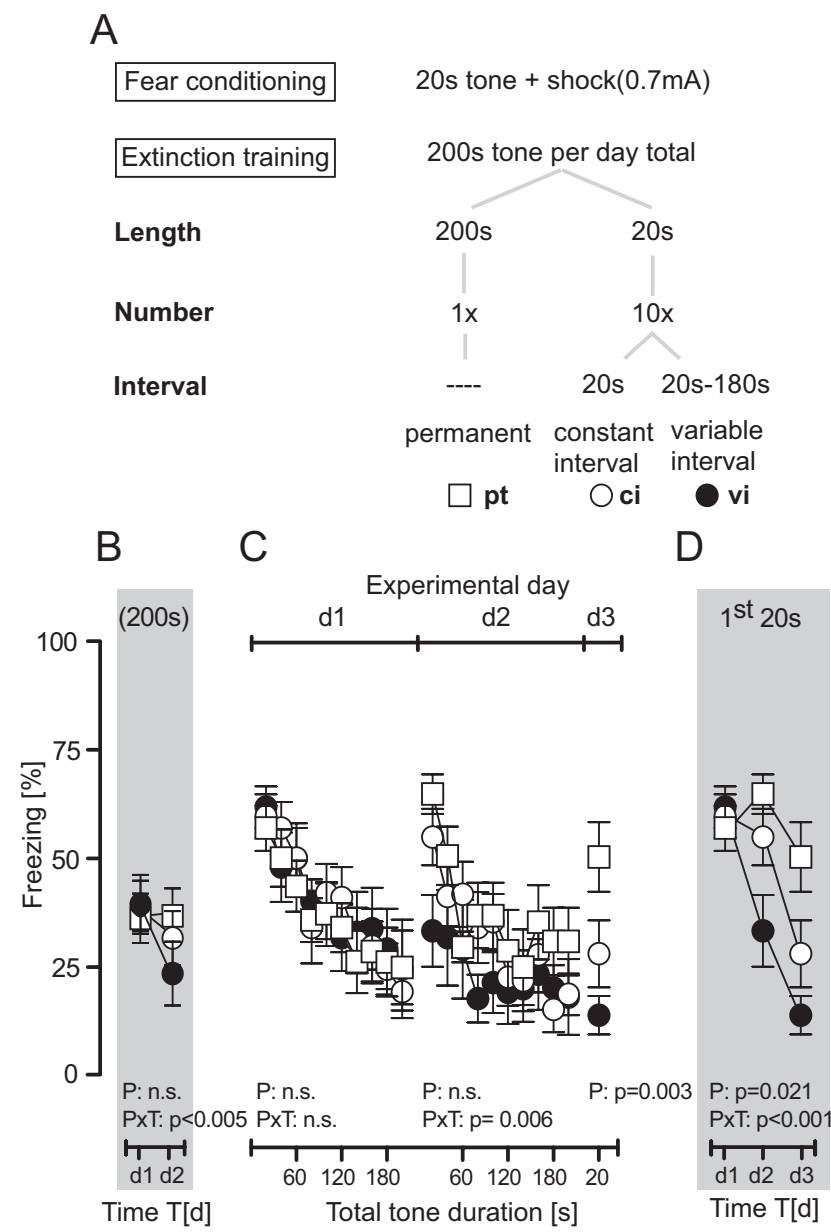

Figure 2. Extinction training as a function of stimulus length, repetition, and predictability. $\boldsymbol{A}$, Experimental schedule depicting that $\mathrm{B} 6 \mathrm{~N}$ mice received a single tone-shock pairing at d0 and were subsequently exposed to one of three tone presentation protocols at $\mathrm{d} 1$ and $\mathrm{d} 2$, followed by a final 20 s tone presentation at $\mathrm{d} 3$ ( $n=10$ per group). $\boldsymbol{B}$, Development of the total freezing responses over the course of repeated extinction training. C, Development of the freezing responses over the course of tone presentations per day (note that freezing to the 200 s tone was analyzed in $20 \mathrm{~s}$ bins). $\boldsymbol{D}$, Development of the freezing responses to the initial $20 \mathrm{~s}$ of tone presentations per day. The duration of freezing was normalized to respective observation periods and presented as the mean \pm SEM. Results of the two-way ANOVA for repeated measurements with the main factors protocol $(P)$ and time $(T)$ are shown in the graphs (for the sake of clarity, we do not report the results of post hoc analyses).

initial freezing responses in the vi group (protocol: $F_{(1,14)}=$ $22.15, p<0.001$; protocol $\times$ time: $\left.F_{(2,28)}=4.69, p=0.017\right)($ Fig. $3 C)$. Mice of the pt group failed to develop between-session extinction despite prominent within-session extinction at day 41 (Fig. $3 B, C$ ). Thus, within-session extinction is also not sufficient for initiating between-session extinction in cases of remote fear memories. Moreover, the fact that the initial freezing response decreased in the vi group from day 40 to day 41 in the absence of within-session extinction at day 40 demonstrates that withinsession extinction is not necessary for initiating betweensession extinction.

\section{CB1 receptor signaling mediates within-session but not} between-session extinction

To further validate our conclusions, we studied fear extinction in mice with abolished CB1 receptor signaling, an animal model of severe impairments in within-session extinction. In agreement with previous observations (Kamprath et al., 2006, 2009), both 


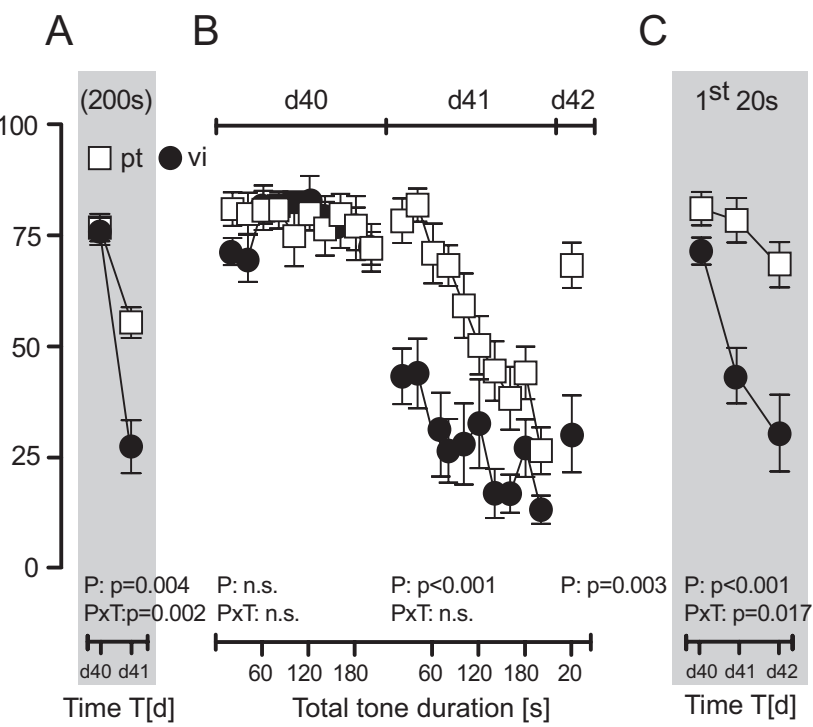

Figure 3. Extinction training after fear incubation. B6N mice received a single tone-shock pairing at $\mathrm{d} 0$ and were exposed to either a pt of $200 \mathrm{~s}$ duration $(n=9)$ or ten $20 \mathrm{~s}$ tones with vi $(n=7)$ at $d 40$ and $d 41$. At $d 42$, all mice were exposed to a single $20 \mathrm{~s}$ tone. $A$, Development of the total freezing responses over the course of repeated extinction training. $\boldsymbol{B}$, Development of the freezing responses over the course of tone presentations per day. $\boldsymbol{C}$, Development of the freezing responses to the initial $20 \mathrm{~s}$ of tone presentations per day. Data were normalized to the respective observation interval and expressed as means \pm SEM. Results of the two-way ANOVA for repeated measurements with the main factors protocol $(P)$ and time $(T)$ are shown in the graphs (for the sake of clarity, we do not report the results of post hoc analyses).

genetic (genotype $\times$ time: $\left.F_{(9,189)} \geq 1.92, p \leq 0.05\right)($ Fig. $4 B)$ and pharmacological prevention of $\mathrm{CB} 1$ receptor signaling (treatment: $\left.F_{(1,19)}>7.47, p<0.01\right)$ (Fig. $4 E$ ) rendered conditioned mice unable to show within-session extinction over the course of a permanent tone presentation. Between-session extinction was considerably more pronounced in wild-type than in CB1deficient mice if the development of the total freezing responses was considered (time: $F_{(9,84)}=10.05, p<0.001$; genotype: $F_{(1,21)}$ $=6.16, p=0.021$; two-way ANOVA) (Fig. $4 A$ ). Also in vehicletreated controls, the total freezing response was significantly smaller than in $\mathrm{B} 6 \mathrm{~N}$ mice treated with the $\mathrm{CB} 1$ receptor antagonist SR141716 (treatment: $F_{(1,19)}=21.37, p<0.001$ ) (Fig. $4 D$ ). In contrast to development of the total freezing responses, we failed to observe any significant decrease in the initial freezing responses to the tone, regardless of the genotype (genotype: $F_{(1,21)}$ $=1.03, p=0.320$; time: $F_{(9,84)}=1.68, p=0.160$; genotype $\times$ time: $F_{(9,84)}=1.30, p=0.273$ ) (Fig. $4 C$ ) or treatment (treatment: $F_{(1,19)}=7.36, p=0.013$; time: $F_{(4,76)}=1.84, p=0.129$; treatment $\times$ time: $\left.F_{(4,76)}=0.99, p=0.413\right)($ Fig. $4 F)$ of the animals. These data corroborate our previous findings that within-session extinction is not sufficient for between-session extinction even under circumstances of more intensified extinction training.

Genetic deletion or pharmacological blockade of CB1 also prevented within-session extinction in the case of extinction training by repeated 20 s tone presentations at variable intervals (day $1: F_{(9,135)}=$ $1.43, p=0.179$, in the case of $\mathrm{CB}^{-1-}$ mice; day 1 and day 2 : $F_{(9,81)}<1.98, p>0.05$, in the case of treatment with SR141716), except for day 2 in $\mathrm{CB} 1^{-1-}$ mice, when within-session extinction was attenuated but not abolished $\left(F_{(9,135)}=2.10, p=0.038\right.$, one-way ANOVAs performed separately per group) (Fig. $5 B, E$ ). In contrast, wild-type and vehicle-treated controls showed prominent within-session extinction at day 1 and day $2\left(F_{(9,144)}>9.69\right.$, $p<0.0001$, in the case of $\mathrm{CB} 1^{+/+}$mice; $F_{(9,99)}>3.62, p<0.001$, in the case of vehicle treatment), before they reached floor levels of freezing behavior (d3). Accordingly, freezing responses were always significantly more pronounced in wild-type (genotype: $F_{(1,31)}>5.93, p<0.020, \mathrm{~d} 1, \mathrm{~d} 2, \mathrm{~d} 3$, and d40) (Fig. 5B) and vehicle-treated mice (treatment: $F_{(1,20)}>9.89, p<0.005$, d1 to d40) (Fig. $5 E$ ). Despite the impairments in within-session extinction, genetic deletion of CB1 failed to affect between-session extinction. This became evident if the development of the total freezing responses (genotype: $F_{(1,31)}=12.13, p=0.001$; time: $F_{(4,124)}=111.5, p<0.001$; genotype $\times$ time: $F_{(4,124)}=0.89, p=$ 0.470 ) (Fig. $5 A$ ) or the initial freezing responses (genotype: $F_{(1,31)}=$ 3.44, $p=0.072$; time: $F_{(4,124)}=74.98, p<0.001$; genotype $\times$ time: $F_{(4,124)}=1.89, p=0.115$ ) (Fig. $5 C$ ) were considered. Essentially, the same was the case after pharmacological blockade of CB1 (Fig. $5 D, F)$ (statistics not shown). All animals showed spontaneous recovery of the freezing response from day 10 to day 40, which was more pronounced in antagonist-treated mice, if development of the total freezing response was considered (treatment $\times$ time: $F_{(4,72)}=$ 3.81, $p=0.007$ ) (Fig. 5D). Together, these data demonstrate that $\mathrm{CB} 1$ receptors play an important role in within-session extinction not only during exposure to permanent tones, but also with repeated tone presentations at variable intervals, without affecting betweensession extinction. This strengthens our previous conclusion that within-session extinction is neither necessary nor sufficient for between-session extinction.

\section{Neuroanatomical basis of relearning}

By combining pharmacological blockade of CB1 receptors with the pt and vi protocols, we ought to dissociate anatomical substrates of within-session and between-session extinction. In agreement with our previous observations, a three-way ANOVA (protocol, drug, time) for repeated measures (time) confirmed that mice failed to show within-session extinction after treatment with SR141716, whereas vehicle-treated controls showed a rapid decline in freezing (drug: $F_{(1,36)}=98.88, p<0.001$; drug $\times$ time: $\left.F_{(9,324)}=34.51, p<0.001\right)$, with no differences between the two extinction protocols (protocol: $F_{(1,36)}=0.043, p=0.835$; protocol $\times$ drug: $F_{(1,36)}=0.044, p=0.834$; protocol $\times$ time: $F_{(9,324)}=$ $1.33, p=0.216$; protocol $\times$ drug $\times$ time: $\left.F_{(9,324)}=1.19, p=0.300\right)$ (Fig. 6, insets). Freezing behavior before tone presentations was negligible and unaffected by pharmacological treatment (Fig. 6, insets) (statistics not shown).

Consequences of permanent versus repeated tone presentations and pharmacological treatment on neuronal activity were assessed by counting the number of c-Fos-immunoreactive neurons (Fos-ir). We focused our attention on those brain areas that are known to be involved in fear and relearning processes, namely, the hippocampal formation, the amygdala complex, and the prefrontal cortex (Singewald, 2007). Two-way ANOVAs with the factors protocol (presentation of permanent tone vs repeated tone presentations vs home-cage controls) and treatment (SR141716 vs vehicle) revealed highly significant main effects of protocol for most of the brain structures under study $\left(F_{(2,27)}>\right.$ $3.84, p<0.05$ ) (Fig. 6C). Post hoc analyses failed to detect any significant differences in Fos-ir between mice exposed to the permanent tone and home-cage controls, except for the CA3 region of the hippocampus and the lateral amygdala (Fig. 6A,B). Repeated exposure to $20 \mathrm{~s}$ tones, in contrast, led to a significant increase in Fos-ir in the dentate gyrus, the basolateral and lateral amygdala, the cingulate cortex, and the prelimbic cortex, compared with mice exposed to a single $200 \mathrm{~s}$ tone and to home-cage controls. As indicated by the lack of protocol $\times$ treatment interactions in most of the brain structures $\left(F_{(2,27)}<2.17, p>0.133\right.$, 
except for IL) (Fig. 6C), these activity patterns were largely independent of treatment with the $\mathrm{CB} 1$ receptor antagonist. However, pharmacological blockade of CB1 led to a general increase in Fos-ir within the CA3 region (weak effect; $F_{(1,27)}=$ 5.98, $p=0.021$ ), lateral amygdala, central amygdala, and prelimbic cortex (strong effects; $F_{(1,27)}>21.7, p<0.001$ ) (Fig. $6 \mathrm{~A}, \mathrm{~B})$, independently of the protocol used (i.e., even in home-cage controls). Only in the case of the infralimbic cortex was there a significant interaction between protocol and treatment $\left(F_{(2,27)}=16.95, p<\right.$ $0.001)$. Whereas vehicle-treated mice with repeated tone presentation showed increased Fos-ir compared with the two other groups, this difference was abolished by treatment with the $\mathrm{CB} 1$ receptor antagonist. Together, freezing to the tone in vehicle-treated mice and precipitation of exaggerated freezing by pharmacological blockade of CB1 failed to reveal significant differences in Fos-ir between mice exposed to the permanent tone and respective home-cage controls. Consequently, expression of freezing per se was not reflected by Fos-ir in the brain structures under study. Repeated tone presentations at variable intervals led to a significant increase in Fos-ir in a number of brain structures, compared with mice exposed to a permanent tone. In the dentate gyrus, basolateral amygdala, and cingulate cortex, these differences were largely independent of the pharmacological blockade of $\mathrm{CB} 1$ and within-session extinction of freezing behavior, suggesting those brain structures as primary places of betweensession extinction. Finally, some brain structures, such as the prelimbic cortex and central amygdala, showed a general increase in Fos-ir on pharmacological blockade of CB1 even in home-cage controls, thus indicating their general involvement in processing of stressful encounters (e.g., injection procedure).

\section{Discussion}

The present study investigated the interrelation between withinsession and between-session extinction in an auditory-cued fearconditioning paradigm. We showed that within-session extinction was the same for permanent and spaced tone presentations. It required intact CB1 signaling and was neither sufficient nor necessary for initiating between-session extinction. Betweensession extinction, in contrast, depended on the pattern and predictability of tone presentations. It was maintained in mice with disrupted CB1 receptor signaling and developed independently of within-session extinction. The dissociation of within- and between-session extinction could also be seen at the neuroanatomical level in terms of c-Fos expression.

The striking similarities in within-session extinction in response to permanent $(\mathrm{pt})$ and spaced (ci and vi) tone presentations at day 1 indicate that the decline in freezing over the course the results of post hoc analyses).
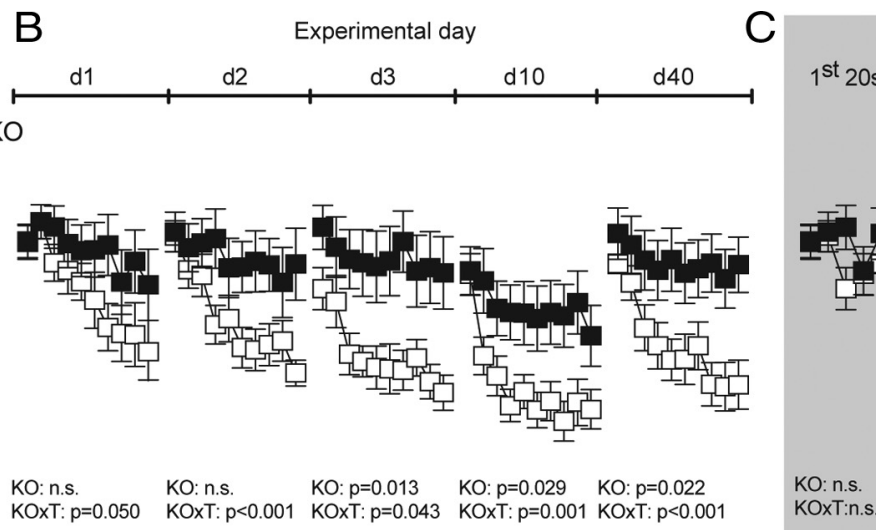

$E$

\section{$F$}

KO: ns. KOxT:n.s.

$\mathrm{F}$
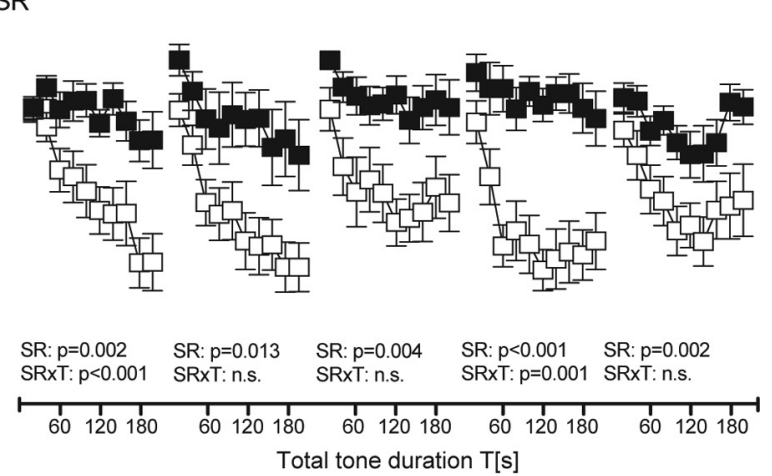

SR: $p=0.013$ SRxT: n.s.

PTTT Time T[d]

Figure 4. Role of $\mathrm{CB} 1$ in extinction training with permanent tones. All animals received a single tone-shock pairing at $\mathrm{d} 0$ olished by either genetic means $(\boldsymbol{A}-\boldsymbol{C})$ or the CB1 receptor antagonist SR141716 $(3 \mathrm{mg} / \mathrm{kg}$, s.c.), which was administered 政 $1 \mathrm{~h}$ before tone presentations at $\mathrm{d} 1$ to $\mathrm{d} 40(\boldsymbol{D}-\boldsymbol{F})$. Analysis of the development of the total freezing responses abse an interval and expressed as means \pm SEM. Results of the two-way ANOVA for repeated measurements with the main factors genotype (KO), treatment (SR), and time (T) are shown in the graphs (for the sake of clarity, we do not report

of a given extinction session likely relates to the total duration and not to the pattern of tone presentation. It might result from the same short-term habituation processes (Kamprath and Wotjak, 2004). Only spaced tone presentations led to between-session extinction. Apparently, repeated experience of the tone-noshock association (vi and ci) is more efficient in establishing an inhibitory memory trace than a single tone presentation per day (pt). Consequently, the absence of the predicted punishment rather than acute fear relief per se seems to cause the formation of a CS-no-US association.

Repeated tone presentations at vi were more efficient in eliciting between-session extinction than tone presentations at ci. This might be explained by the role of attention in extinction learning. For instance, some authors propose that focusing attention on the fear stimulus favors fear reduction (Kamphuis and Telch, 2000), which, however, is disputed by others (Oliver and Page, 2003). In the present study, unexpected stimulus presentation may raise attention toward the tone, whereas tone presentations at regular intervals may favor stimulus expectancy and, thus, reduce attention. In this context, it is of note that temporal predictability could promote habituation of neuronal activity in brain structures such as the lateral and basolateral amygdala 


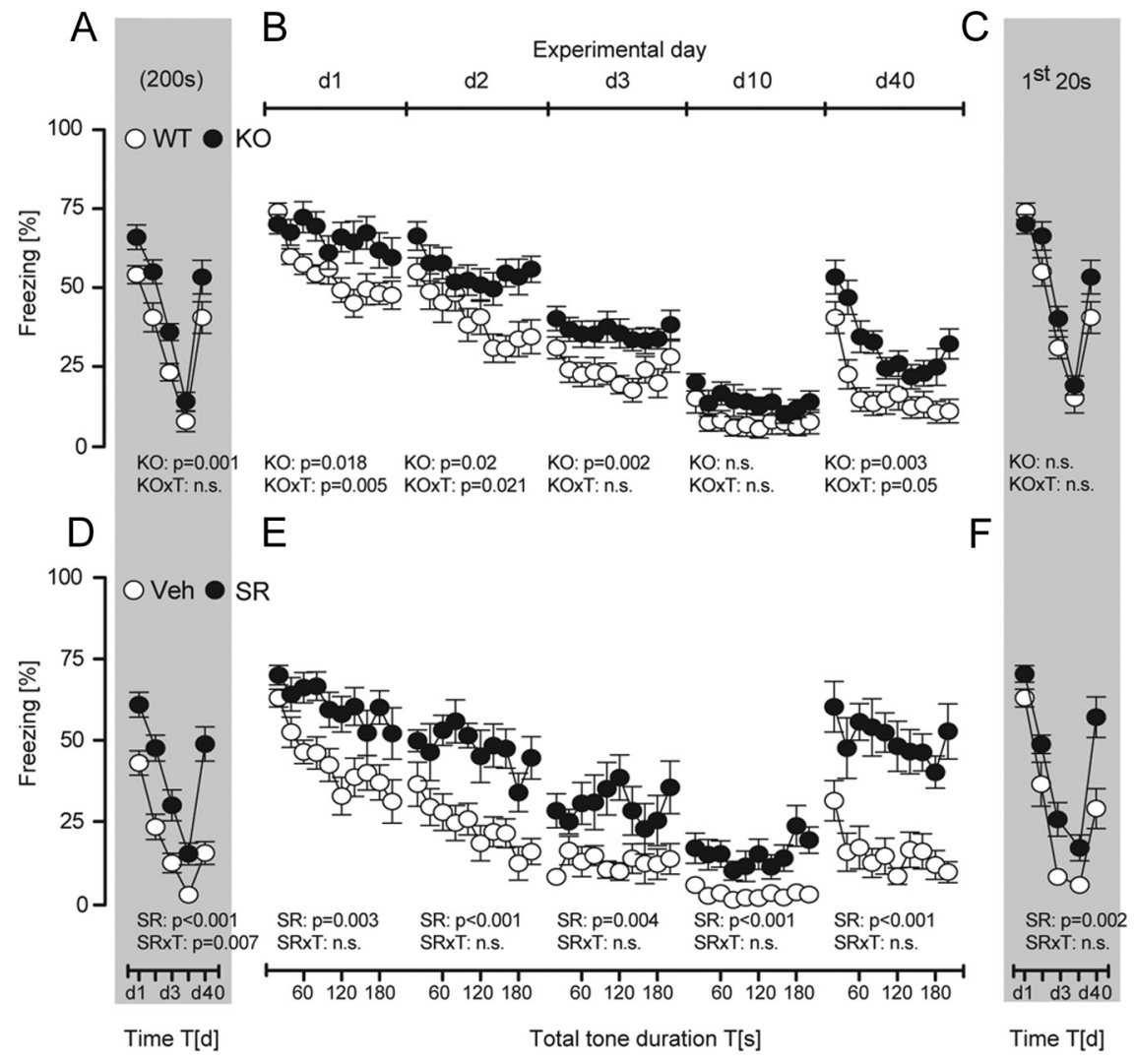

Figure 5. Role of $C B 1$ in extinction training with repeated tone presentations at variable intervals. All animals received a single tone-shock pairing at $\mathrm{d} 0$ and were subsequently exposed to ten $20 \mathrm{~s}$ tones at vi (compare Fig. $2 A$ ) from d1 to d40. A-F, CB1 receptor signaling was abolished either by genetic means $(A-C)$ or by the $(B 1$ receptor antagonist $S R 141716(3 \mathrm{mg} / \mathrm{kg}$, s.c.), which was administered to B6N mice $1 \mathrm{~h}$ before the first tone presentation at $\mathrm{d} 1$ to $\mathrm{d} 40(\boldsymbol{D}-\boldsymbol{F})$. Analysis of the development of the total freezing responses over the course of repeated extinction training $(A, D)$, the development of the freezing responses over the course of tone presentations per day $(\boldsymbol{B}, \boldsymbol{E})$, and the development of the freezing responses to the first $20 \mathrm{~s}$ tone per day $(\boldsymbol{C}, \boldsymbol{F})$ of CB1-deficient mice (K0; $n=16)$, wild-type littermates (WT; $n=17)$, and B6N mice with pharmacological blockade of CB1 (SR, $n=10)$ or vehicle treatment $(n=12)$. Data were normalized to the respective observation interval and expressed as means \pm SEM. Results of the two-way ANOVA for repeated measurements with the main factors genotype (KO), treatment (SR), and time (T) are shown in the graphs (for the sake of clarity, we do not report the results of post hoc analyses).

(Herry et al., 2007), which had been implicated in extinction learning (Falls et al., 1992; Herry et al., 2008).

Fear incubation for several weeks led to a sustained freezing response to the tone. Other than for contextual fear memory (Woods and Bouton, 2008), little is known about the dependency of fear extinction on the age of cued fear memories. The only exceptions are studies investigating consequences of extinction training initiated early after conditioning (e.g., Myers et al., 2006; Kim and Richardson, 2009). Here, we demonstrate that mice showed decaying freezing $1 \mathrm{~d}$ after but sustained freezing $40 \mathrm{~d}$ after the tone-shock pairing, regardless of the tone presentation protocol (pt vs vi). Contextual fear memory is known to undergo major reorganization between recent and remote stages (Frankland et al., 2004). For instance, remote contextual memories are stored in distributed cortical networks (Woods and Bouton, 2008). It remains to be shown whether auditorycured fear memory undergoes similar changes and whether these changes are the cause for the less efficient within-session extinction at late time points after conditioning.

Despite the complete absence of within-session extinction at day 40 , tone presentation at variable intervals readily elicited between-session extinction, thus indicating that within-session extinction is not only insufficient (as discussed before), but also dispensable for initiation of between-session extinction. This finding points to an uncoupling of fear expression and cognitive appraisal of changes in CS-US contingencies, which is supported by the results obtained in mice with genetic deletions or pharmacological blockade of CB1 receptors. Those interventions abolished or severely attenuated within-session extinction but failed to affect between-session extinction triggered by repeated tone presentations at variable intervals. The phenotype seen in withinsession extinction precludes that the lack of involvement of CB1 in between-session extinction relates to inefficient activation of the endocannabinoid system (Kamprath et al., 2009). It rather implies that CB1 signaling is dispensable for relearning of the CS-US contingency, similarly to the situation in appetitive conditioning tasks (Hölter et al., 2005). This conclusion seemingly contradicts numerous studies reporting a significant role of the endocannabinoid system in between-session extinction (Viveros et al., 2007; Moreira and Lutz, 2008). To resolve this issue, we propose that extinction of elemental fear memories follows different rules than extinction of contextual/hippocampusdependent memories. Furthermore, the impairments of CB1-deficient mice in between-session extinction of auditorycued fear memories reported before (Marsicano et al., 2002) might be explained by an involvement of the endocannabinoid system in habituation to homotypic stressors (Patel and Hillard, 2008). Repeated confrontation with the same aversive encounter is accompanied by increased endocannabinoid signaling, which facilitates acute habituation to the stressor (Patel et al., 2005b). A similar scenario may apply to fear extinction (Kamprath et al., 2006), where group differences between CB1deficient mice and wild-type controls became stronger on repeated tone presentation (Fig. $4 B$ ). This accelerated withinsession extinction seemingly results in between-session extinction, if development of the total freezing responses is considered (Fig. 4A), but does not affect the initial freezing responses per day (Fig. 4C). Our failure to observe a similar acceleration of withinsession extinction in vehicle-treated controls (Fig. 4D) might be explained by summative effects of exposure to the tone and stressful injection procedure, which may result in an almost maximal activation of the endocannabinoid system right from the beginning of extinction training.

If extinction training indeed leads to relearning of the toneshock contingency and the formation of a new inhibitory toneno-shock association that suppresses the expression of the original memory trace (Myers and Davis, 2002, 2007; Ehrlich et al., 2009), we should expect decreased fear responses right from the beginning of the tone presentation. Therefore, we propose that the development of the initial fear responses to the tone over the course of repeated extinction training represents the most direct measure of between-session extinction. This measure is superior to analysis of total fear responses, since it is not con- 

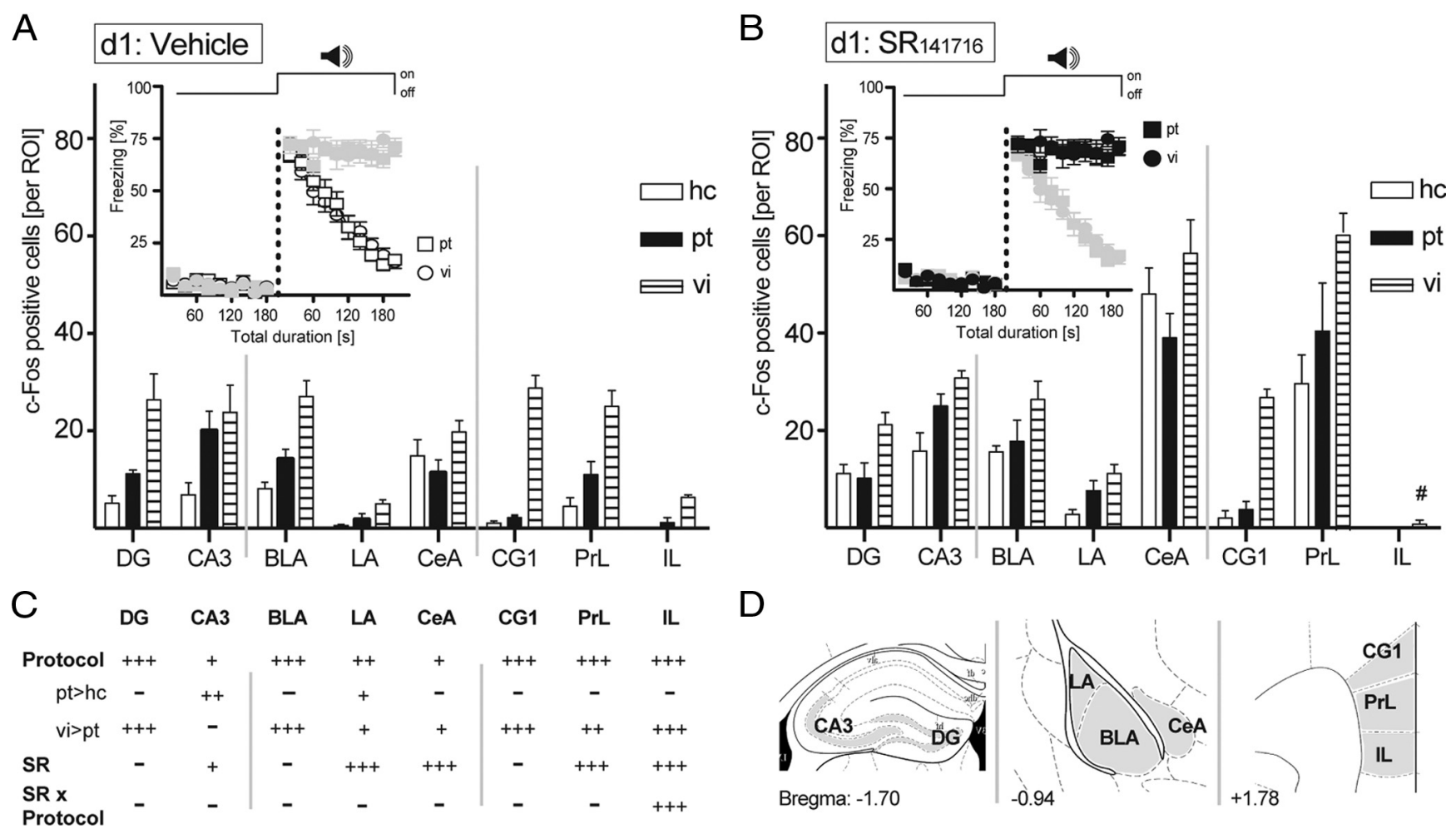

Figure 6. Neuroanatomical signature of extinction training. B6N mice received a single tone-shock pairing at d0 and were exposed to either a pt of $200 \mathrm{~s}$ duration (compare Fig. $2 \mathrm{~A}$ ) or to ten $20 \mathrm{~s}$ tones at vi (compare Fig. $2 A)$, or stayed in their $\mathrm{hc} 24 \mathrm{~h}$ later (d1). $\boldsymbol{A}, \boldsymbol{B}$, Half of the animals of each group were treated with vehicle $(\boldsymbol{A})$, and the other half were treated with the $C B 1$ receptor antagonist SR141716 (3 mg/kg) $(\boldsymbol{B}) 1 \mathrm{~h}$ before tone presentation (in the case of pt and vi). All animals were killed $70 \mathrm{~min}$ after the end of the exposure (or after the same time in the home cage), and the brains were processed for c-Fos immunohistochemistry $(\boldsymbol{A}, \boldsymbol{B})$. Insets show the development of the freezing responses before and during tone presentations, whereby freezing data of the animals of the same treatment groups as used for immunohistochemistry in the respective figure panel are shown in black and those of the other treatment groups in gray. $C$, Results of the two-way ANOVAs with the main factors protocol (P) and treatment (SR), performed separately per brain structure $\left({ }^{-} p>0.05 ;{ }^{+} p<0.05 ;{ }^{++} p<0.01 ;{ }^{+++} p<0.001\right.$; ${ }^{\#}$ significant SR-protocol interaction). D, The total number of c-Fos-positive neurons was counted bilaterally in the ROIs in one section per mouse. ROIs were defined according to the mouse brain atlas (Franklin and Paxinos, 1997; compare Fig. 1). Values are expressed as the mean $\pm \operatorname{SEM}(n=5$ per group).

founded by differences in within-session extinction (as discussed before). Accordingly, the reoccurrence of the freezing response with the passage of time (i.e., spontaneous recovery) should be assessed by means of the initial fear responses rather than the total fear responses. In any case, comparisons of freezing responses shown at the end of an extinction session and the beginning of the next have to be avoided because of the apparent independence of within-session and between-session extinction revealed by the present study.

We could dissociate within-session and between-session extinction not only at the behavioral and molecular levels (in terms of CB1 signaling), but also at the anatomical level. Exposure to tones at variable intervals caused increased Fos-ir in the lateral and basolateral amygdala, compared to mice with exposure to permanent tones and to home-cage controls. A similar pattern of Fos-ir was observed in the dentate gyrus, the cingulate cortex, and the prelimbic cortex. However, only in the basolateral amygdala, dentate gyrus, and cingulate cortex, Fos-ir was largely independent of pharmacological blockade of CB1 receptors and/or differences in expression of conditioned fear. Given the fact that between-session extinction was unaffected by impairments in CB1 signaling (Fig. 5C,F), these three brain structures might represent hot spots for relearning processes, which involve plastic changes in neuronal activity. This conclusion is supported by pharmacological studies reporting, for instance, that local inhibition of NMDA receptors (Falls et al., 1992) or mitogenactivated protein kinase activity (Herry et al., 2006) within the basolateral amygdala impairs between-session extinction. Recently, Hefner et al. (2008) performed a thorough investigation of the neuronal matrix underlying extinction recall. They studied Fos-ir in extinction-prone and extinction-resistant mouse strains and reported significant strain differences primarily within the basolateral amygdala and the infralimbic cortex. Consequently, the formation of a new tone-no-shock association and recall of this inhibitory memory trace seems to involve, at least in part, the same brain structures.

Pharmacological blockade of CB1 led to a large increase in Fos-ir primarily within the central amygdala and prelimbic cortex, regardless of the exposure protocol, i.e., even in home-cage controls. These data provide compelling evidence that not only freezing responses elicited by electrical stimulation or pharmacological manipulation of distinct brain structures (Vianna et al., 2003; Borelli et al., 2006), but also expression of conditioned fear fails to induce c-Fos expression in a variety of brain structures known to be implicated in fear and anxiety (Singewald, 2007). Other markers of neuronal activity, such as phosphorylation of kinases, may better reflect differences in freezing behavior (in particular if analyzed in the absence of injection stress in CB1-KO and CB1-WT mice) (Cannich et al., 2004). Previous studies reported an increase in Fos-ir in the central amygdala of naive mice following pharmacological blockade of CB1 (Patel et al., 2005a). In addition, $\mathrm{CB} 1$ plays a complex role in the regulation of fear and anxiety within the medial prefrontal cortex (Lin et al., 2009), and neuronal activity within the prelimbic cortex seems to directly 
relate to acute fear expression (Vidal-Gonzalez et al., 2006; Burgos-Robles et al., 2009). Therefore, the most parsimonious interpretation of our data is that c-Fos expression at the level of the CeA and PrL reflects impaired endocannabinoid signaling, likely because of exaggerated stress responses and discomfort resulting from the injection procedure (Steiner and Wotjak, 2008). Freezing performance per se is not mirrored by c-Fos. At the level of the BLA, DG, and Cg1, finally, increased c-Fos expression reflects relearning of tone-shock contingencies (i.e., between-session extinction), independently of the acute freezing response (i.e., within-session extinction) and/or disturbed CB1 receptor functioning.

The infralimbic cortex was the only brain structure where we observed a significant interaction between tone presentation and antagonist treatment. Induction of between-session extinction by repeated exposure to tones at variable intervals caused a significant increase in Fos-ir within that brain structure, compared with home-cage controls and exposure to a permanent tone. Pharmacological blockade of CB1 abolished these effects. This is a striking observation, since the infralimbic cortex undergoes plastic changes during relearning/extinction training (Santini et al., 2008), which are thought to underlie its subsequent involvement in suppression of conditioned fear during extinction recall (Milad and Quirk, 2002). However, in light of the largely intact between-session extinction observed in mice with pharmacological blockade of CB1 (Fig. 5F), attenuation of Fos expression following treatment with SR141716 might be of minor biological significance for the behavioral phenotype in the present study. It remains to be shown to what extent the differences in Fos-ir in the infralimbic cortex contribute to the reduced retention of fear extinction observed in these animals if alterations in spontaneous recovery of freezing from day 10 to day 40 are considered (Fig. $5 D, F)$.

Together, our data reveal an uncoupling between the expression of conditioned fear and the relearning of tone-shock contingencies at molecular, anatomical, and behavioral levels. This asks for reconsideration of current strategies in exposure-based therapies in human subjects. As proposed by Craske et al. (2008), within-session fear reduction does not predict therapy success. The apparent dissociation of emotional and cognitive processing disproves exposure paradigms in which the length of an exposure session is defined by the time point of maximal acute fear relief. Protocols should rather favor fast and effective buildup of inhibitory memory traces regardless of acute fear levels. Pharmacological prolongation of endocannabinoid signaling (Di Marzo, 2008) may not directly affect the inhibitory learning process, but may reduce the emotional load during an exposure session and, thus, increase compliance rates.

\section{References}

Barad M (2005) Fear extinction in rodents: basic insight to clinical promise. Curr Opin Neurobiol 15:710-715.

Borelli KG, Ferreira-Netto C, Brandão ML (2006) Distribution of Fos immunoreactivity in the rat brain after freezing or escape elicited by inhibition of glutamic acid decarboxylase or antagonism of GABA-A receptors in the inferior colliculus. Behav Brain Res 170:84-93.

Burgos-Robles A, Vidal-Gonzalez I, Quirk GJ (2009) Sustained conditioned responses in prelimbic prefrontal neurons are correlated with fear expression and extinction failure. J Neurosci 29:8474-8482.

Cannich A, Wotjak CT, Kamprath K, Hermann H, Lutz B, Marsicano G (2004) CB1 cannabinoid receptors modulate kinase and phosphatase activity during extinction of conditioned fear in mice. Learn Mem 11:625-632.

Craske MG, Kircanski K, Zelikowsky M, Mystkowski J, Chowdhury N, Baker A
(2008) Optimizing inhibitory learning during exposure therapy. Behav Res Ther 46:5-27.

Di Marzo V (2008) Targeting the endocannabinoid system: to enhance or reduce? Nat Rev Drug Discov 7:438-455.

Ehrlich I, Humeau Y, Grenier F, Ciocchi S, Herry C, Lüthi A (2009) Amygdala inhibitory circuits and the control of fear memory. Neuron 62:757-771.

Falls WA, Miserendino MJ, Davis M (1992) Extinction of fear-potentiated startle: blockade by infusion of an NMDA antagonist into the amygdala. J Neurosci 12:854-863.

Frankland PW, Bontempi B, Talton LE, Kaczmarek L, Silva AJ (2004) The involvement of the anterior cingulate cortex in remote contextual fear memory. Science 304:881-883.

Franklin K, Paxinos G (1997) The mouse brain in stereotaxic coordinates. San Diego: Academic.

Grös DF, Antony MM (2006) The assessment and treatment of specific phobias: a review. Curr Psychiatry Rep 8:298-303.

Hefner K, Whittle N, Juhasz J, Norcross M, Karlsson RM, Saksida LM, Bussey TJ, Singewald N, Holmes A (2008) Impaired fear extinction learning and cortico-amygdala circuit abnormalities in a common genetic mouse strain. J Neurosci 28:8074-8085.

Herry C, Trifilieff P, Micheau J, Lüthi A, Mons N (2006) Extinction of auditory fear conditioning requires MAPK/ERK activation in the basolateral amygdala. Eur J Neurosci 24:261-269.

Herry C, Bach DR, Esposito F, Di Salle F, Perrig WJ, Scheffler K, Lüthi A, Seifritz E (2007) Processing of temporal unpredictability in human and animal amygdala. J Neurosci 27:5958-5966.

Herry C, Ciocchi S, Senn V, Demmou L, Müller C, Lüthi A (2008) Switching on and off fear by distinct neuronal circuits. Nature 454:600-606.

Hölter SM, Kallnik M, Wurst W, Marsicano G, Lutz B, Wotjak CT (2005) Cannabinoid CB1 receptor is dispensable for memory extinction in an appetitively-motivated learning task. Eur J Pharmacol 510:69-74.

Kamphuis JH, Telch MJ (2000) Effects of distraction and guided threat reappraisal on fear reduction during exposure-based treatments for specific fears. Behav Res Ther 38:1163-1181.

Kamprath K, Wotjak CT (2004) Nonassociative learning processes determine expression and extinction of conditioned fear in mice. Learn Mem 11:770-786.

Kamprath K, Marsicano G, Tang J, Monory K, Bisogno T, Di Marzo V, Lutz B, Wotjak CT (2006) Cannabinoid CB1 receptor mediates fear extinction via habituation-like processes. J Neurosci 26:6677-6686.

Kamprath K, Plendl W, Marsicano G, Deussing JM, Wurst W, Lutz B, Wotjak CT (2009) Endocannabinoids mediate acute fear adaptation via glutamatergic neurons independently of corticotropin-releasing hormone signaling. Genes Brain Behav 8:203-211.

Kim JH, Richardson R (2009) Expression of renewal is dependent on the extinction-test interval rather than the acquisition-extinction interval. Behav Neurosci 123:641-649.

Lin HC, Mao SC, Su CL, Gean PW (2009) The role of prefrontal cortex CB1 receptors in the modulation of fear memory. Cereb Cortex 19:165-175.

Marks I, Tobena A (1990) Learning and unlearning fear: a clinical and evolutionary perspective. Neurosci Biobehav Rev 14:365-384.

Marsicano G, Wotjak CT, Azad SC, Bisogno T, Rammes G, Cascio MG, Hermann H, Tang J, Hofmann C, Zieglgänsberger W, Di Marzo V, Lutz B (2002) The endogenous cannabinoid system controls extinction of aversive memories. Nature 418:530-534.

McSweeney FK, Swindell S (2002) Common processes may contribute to extinction and habituation. J Gen Psychol 129:364-400.

Milad MR, Quirk GJ (2002) Neurons in medial prefrontal cortex signal memory for fear extinction. Nature 420:70-74.

Moreira FA, Lutz B (2008) The endocannabinoid system: emotion, learning and addiction. Addict Biol 13:196-212.

Myers KM, Davis M (2002) Behavioral and neural analysis of extinction. Neuron 36:567-584

Myers KM, Davis M (2007) Mechanisms of fear extinction. Mol Psychiatry 12:120-150.

Myers KM, Ressler KJ, Davis M (2006) Different mechanisms of fear extinction dependent on length of time since fear acquisition. Learn Mem 13:216-223.

Norton PJ, Price EC (2007) A meta-analytic review of adult cognitivebehavioral treatment outcome across the anxiety disorders. J Nerv Ment Dis 195:521-531. 
Oliver NS, Page AC (2003) Fear reduction during in vivo exposure to bloodinjection stimuli: distraction vs. attentional focus. Br J Clin Psychol 42:13-25.

Patel S, Hillard CJ (2008) Adaptations in endocannabinoid signaling in response to repeated homotypic stress: a novel mechanism for stress habituation. Eur J Neurosci 27:2821-2829.

Patel S, Cravatt BF, Hillard CJ (2005a) Synergistic interactions between cannabinoids and environmental stress in the activation of the central amygdala. Neuropsychopharmacology 30:497-507.

Patel S, Roelke CT, Rademacher DJ, Hillard CJ (2005b) Inhibition of restraint stress-induced neural and behavioural activation by endogenous cannabinoid signalling. Eur J Neurosci 21:1057-1069.

Pitman RK, Orr SP, Altman B, Longpre RE, Poiré RE, Macklin ML (1996a) Emotional processing during eye movement desensitization and reprocessing therapy of Vietnam veterans with chronic posttraumatic stress disorder. Compr Psychiatry 37:419-429.

Pitman RK, Orr SP, Altman B, Longpre RE, Poiré RE, Macklin ML, Michaels MJ, Steketee GS (1996b) Emotional processing and outcome of imaginal flooding therapy in Vietnam veterans with chronic posttraumatic stress disorder. Compr Psychiatry 37:409-418.

Richter K, Wolf G, Engelmann M (2005) Social recognition memory requires two stages of protein synthesis in mice. Learn Mem 12:407-413.

Rinaldi-Carmona M, Barth F, Héaulme M, Shire D, Calandra B, Congy C, Martinez S, Maruani J, Néliat G, Caput D (1994) SR141716A, a potent and selective antagonist of the brain cannabinoid receptor. FEBS Lett 350:240-244.
Rosen JB, Schulkin J (1998) From normal fear to pathological anxiety. Psychol Rev 105:325-350.

Santini E, Quirk GJ, Porter JT (2008) Fear conditioning and extinction differentially modify the intrinsic excitability of infralimbic neurons. J Neurosci 28:4028-4036.

Singewald N (2007) Altered brain activity processing in high-anxiety rodents revealed by challenge paradigms and functional mapping. Neurosci Biobehav Rev 31:18-40.

Steiner MA, Wotjak CT (2008) Role of the endocannabinoid system in regulation of the hypothalamic-pituitary-adrenocortical axis. Prog Brain Res 170:397-432.

Vianna DM, Borelli KG, Ferreira-Netto C, Macedo CE, Brandão ML (2003) Fos-like immunoreactive neurons following electrical stimulation of the dorsal periaqueductal gray at freezing and escape thresholds. Brain Res Bull 62:179-189.

Vidal-Gonzalez I, Vidal-Gonzalez B, Rauch SL, Quirk GJ (2006) Microstimulation reveals opposing influences of prelimbic and infralimbic cortex on the expression of conditioned fear. Learn Mem 13:728-733.

Viveros MP, Marco EM, Llorente R, López-Gallardo M (2007) Endocannabinoid system and synaptic plasticity: implications for emotional responses. Neural Plast 2007:52908.

Woods AM, Bouton ME (2008) Immediate extinction causes a less durable loss of performance than delayed extinction following either fear or appetitive conditioning. Learn Mem 15:909-920. 